The Journal of $\mathbf{N}_{\text {onlinear }} \mathbf{S}_{\text {cience and }}$ A pplications

http://www.tjnsa.com

\title{
POSITIVE SOLUTIONS FOR A CLASS OF SINGULAR TWO POINT BOUNDARY VALUE PROBLEMS
}

\author{
RAHMAT ALI KHAN ${ }^{1, *}$ AND NASEER AHMAD ASIF ${ }^{2}$ \\ Communicated by J. J. Nieto
}

\begin{abstract}
Existence of positive solution for a class of singular boundary value problems of the type

$$
\begin{aligned}
& -x^{\prime \prime}(t)=f\left(t, x(t), x^{\prime}(t)\right), \quad t \in(0,1) \\
& x(0)=0, x(1)=0,
\end{aligned}
$$

is established. The nonlinearity $f \in C((0,1) \times(0, \infty) \times(-\infty, \infty),(-\infty, \infty))$ is allowed to change sign and is singular at $t=0, t=1$ and/or $x=0$. An example is included to show the applicability of our result.
\end{abstract}

\section{INTRODUCTION AND PRELIMINARIES}

Singular boundary value problems arise in various fields of Mathematics and Physics such as nuclear physics, boundary layer theory, nonlinear optics, gas dynamics, etc, 11, 5, 9, 12, 14, 17, 18, 19]. For more details on singular BVPs and recent developments, we refer the readers to the recent monograph by R. P. Agarwal and D. O' Regan [4] and [6, 8, 9, 15].

In this paper, we consider a class of second order singular boundary value problems of the type

$$
\begin{aligned}
-x^{\prime \prime}(t) & =f\left(t, x(t), x^{\prime}(t)\right), t \in(0,1), \\
x(0) & =0, x(1)=0,
\end{aligned}
$$

Date: Received: 2 December 2008; Revised: 25 February 2009.

2000 Mathematics Subject Classification. Primary 34A45; Secondary 34B15.

Key words and phrases. Positive solutions, Singular differential equations, Dirichlet boundary conditions.

* Corresponding author. 
where $f \in C((0,1) \times(0, \infty) \times(-\infty, \infty),(-\infty, \infty))$ and may be singular at $t=0$, $t=1$ and/or $x=0$ and is allowed to change sign. We establish existence of positive solutions for the BVP (1.1) under a weaker hypothesis on $f$. Recently, existence of positive solutions for singular boundary value problems, in the case when the nonlinearity $f$ is independent of the derivative term, has been studied by many authors, [3, 10, 11, 13, 21. In these papers, the nonlinearity is assumed to be non-negative and either be sublinear or superlinear. Hence, the results of these papers would be applicable to a limited class of boundary values problems. Moreover, most of nonlinear problems from the applied sciences, the nonlinearity explicitly depends on the derivative term, for example, the differential equation

$$
x^{\prime \prime}(t)=-\lambda\left(\frac{1-t^{2}}{x(t)}\right)^{\prime}-\frac{t}{x(t)}, t \in[0,1),
$$

together with some suitable boundary conditions, that explicitly depends on the derivative, arises in the boundary layer theory in fluid mechanics [19]. Further, the nonlinearity $f$ does not satisfy the sublinear and superlinear conditions in most cases and may change sign. Hence, the study of boundary value problems without the above mentioned restrictions is of great importance.

Recently, existence theory for positive solutions of two point boundary value problems without the first derivative term is studied in [16, 19, 20]. Inspired by the above papers, the aim of the present paper is to improve and generalize the result studied in [20] to the case when the nonlinearity $f$ explicitly depends on the derivative term $x^{\prime}$. We study the problem under much weaker hypothesis on $f$. We include an example to show the applicability of our result.

Throughout this paper, we assume that the following condition holds. $\left(A_{1}\right)$ there exist $k \in C((0,1),(0, \infty))$ and a decreasing $F \in C((0, \infty),(0, \infty))$ such that

$$
\int_{0}^{1} t(1-t) k(t) d t<\infty \text { and } \int_{0}^{\infty} \frac{d u}{F(u)}=\infty .
$$

The condition $\int_{0}^{\infty} \frac{d u}{F(u)}=\infty$ implies that we can choose $R>1$ such that

$$
\int_{1}^{R} \frac{d u}{F(u)}>\max \left\{\int_{0}^{1 / 2} s k(s) d s, \int_{1 / 2}^{1}(1-s) k(s) d s\right\}
$$

For fixed $n \in\{3,4,5, \ldots\}$, let $M=\max \left\{F\left(\frac{1}{n}\right) k(t): t \in\left[\frac{1}{n}, 1-\frac{1}{n}\right]\right\}$ and choose $C>\sqrt{2 M R}$.

For $u \in C[0,1]$ we write $\|u\|=\max \{|u(t)|: t \in[0,1]\}$ and for $u \in C^{1}[0,1]$, we write $\|u\|_{1}=\max \left\{\|x\|, \frac{\gamma}{3}\left\|x^{\prime}\right\|\right\}$ where $\gamma=\frac{n-2}{n}$ for $n \geq 3$. Clearly, $C^{1}[0,1]$ with the norm $\|.\|_{1}$ is a Banach space.

The only condition we are imposing on the nonlinearity $f$ is the following:

$$
0 \leq f\left(t, x(t), x^{\prime}(t)\right) \leq k(t) F(x(t)) \text { on }(0,1) \times(0, R] \times[-C, C] .
$$


For fixed $n \in\{3,4,5, \ldots\}$, consider the BVPs

$$
\begin{aligned}
-x^{\prime \prime}(t) & =f\left(t, x(t), x^{\prime}(t)\right), t \in\left[\frac{1}{n}, 1-\frac{1}{n}\right], \\
x\left(\frac{1}{n}\right) & =\frac{1}{n}, x\left(1-\frac{1}{n}\right)=\frac{1}{n} .
\end{aligned}
$$

We write (1.3) as an equivalent integral equation

$$
x(t)=\frac{1}{n}+\int_{1 / n}^{1-1 / n} G_{n}(t, s) f\left(s, x(s), x^{\prime}(s)\right) d s, t \in\left[\frac{1}{n}, 1-\frac{1}{n}\right],
$$

where

$$
G_{n}(t, s)=\frac{n}{n-2} \begin{cases}\left(s-\frac{1}{n}\right)\left(1-\frac{1}{n}-t\right), & \text { if } \frac{1}{n} \leq s<t \leq 1-\frac{1}{n} \\ \left(t-\frac{1}{n}\right)\left(1-\frac{1}{n}-s\right), & \text { if } \frac{1}{n} \leq t \leq s \leq 1-\frac{1}{n}\end{cases}
$$

is the Green's function for the corresponding homogeneous problem

$$
\begin{aligned}
-x^{\prime \prime}(t) & =0, t \in\left[\frac{1}{n}, 1-\frac{1}{n}\right] \\
x\left(\frac{1}{n}\right) & =0, x\left(1-\frac{1}{n}\right)=0 .
\end{aligned}
$$

Notice that $G_{n}(t, s) \geq 0$ on $\left(\frac{1}{n}, 1-\frac{1}{n}\right) \times\left(\frac{1}{n}, 1-\frac{1}{n}\right)$ and $G_{n}(t, s) \leq G_{n}(s, s), t \in(0,1)$. Moreover,

$$
\begin{array}{r}
\max _{t \in[0,1]} \int_{1 / n}^{1-1 / n} G_{n}(t, s) d s=\int_{1 / n}^{1-1 / n} G_{n}(s, s) d s=\frac{\gamma^{2}}{6}, \\
\max _{t \in[0,1]}\left|\int_{1 / n}^{1-1 / n} \frac{\partial G_{n}}{\partial t}(t, s) d s\right|=\int_{1 / n}^{1-1 / n}\left|\frac{\partial G_{n}}{\partial t}(t, s)\right| d s=\frac{\gamma}{2} .
\end{array}
$$

\section{MAin Results}

Theorem 2.1. Assume that $\left(A_{1}\right)$ and $\left(A_{2}\right)$ hold. Then boundary value problem (1.3) has a solution $x \in C^{1}[0,1]$ such that $\frac{1}{n} \leq x(t)<R$ and $\left|x^{\prime}(t)\right|<C$ for $t \in$ $\left[\frac{1}{n}, 1-\frac{1}{n}\right]$.

Proof. Define retractions $q:(-\infty, \infty) \rightarrow[-C, C]$ by $q(v)=\max \{-C, \min \{v, C\}\}$ and $p:(-\infty, \infty) \rightarrow\left[\frac{1}{n}, R\right]$ by $p_{n}(x(t))=\max \left\{\frac{1}{n}, \min \{x(t), R\}\right\}$. Clearly, $q, p$ are continuous and $q(v)=v$ for $|v| \leq C, p(v)=v$ for $\frac{1}{n} \leq v \leq R$.

Consider the modified BVP

$$
\begin{aligned}
-x^{\prime \prime}(t) & =F_{n}\left(t, x(t), x^{\prime}(t)\right), t \in\left[\frac{1}{n}, 1-\frac{1}{n}\right], \\
x\left(\frac{1}{n}\right) & =\frac{1}{n}, x\left(1-\frac{1}{n}\right)=\frac{1}{n},
\end{aligned}
$$

where $F_{n}\left(t, x(t), x^{\prime}(t)\right)=f\left(t, p_{n}(x(t)), q\left(x^{\prime}(t)\right)\right)$. Clearly, $F_{n}$ is continuous, bounded and nonnegative on $\left[\frac{1}{n}, 1-\frac{1}{n}\right] \times \mathbb{R} \times \mathbb{R}$. Further, any solution $x \in C^{1}[0,1]$ of $(2.1)$ such that

$$
\frac{1}{n} \leq x(t)<R,\left|x^{\prime}(t)\right|<C, t \in\left[\frac{1}{n}, 1-\frac{1}{n}\right],
$$

is a solution of (1.3). Obviously, $x(t) \geq \frac{1}{n}$ on $\left[\frac{1}{n}, 1-\frac{1}{n}\right]$ as $F_{n} \geq 0$. 
We write the BVP 2.1) as an equivalent integral equation of the type

$$
x(t)=\frac{1}{n}+\int_{1 / n}^{1-1 / n} G_{n}(t, s) F_{n}\left(s, x(s), x^{\prime}(s)\right) d s
$$

and define an operator $T_{n}: C^{1}[0,1] \rightarrow C^{1}[0,1]$ by

$$
\left(T_{n} x\right)(t)=\frac{1}{n}+\int_{1 / n}^{1-1 / n} G_{n}(t, s) F_{n}\left(s, x(s), x^{\prime}(s)\right) d s .
$$

By a solution of 2.1 we mean a solution of the operator equation $\left(I-T_{n}\right)(x)=0$, that is, a fixed point of $T_{n}$. We show that $T_{n}$ has a fixed point $x \in C^{1}[0,1]$. Clearly, $T_{n}$ is continuous and completely continuous as $F_{n}$ is continuous and bounded.

Choose $\bar{R}>\max \left\{R, \frac{1}{3}+\frac{M_{1} \gamma^{2}}{6}\right\}$, where

$$
M_{1}=\max \left\{f\left(t, x, x^{\prime}\right): t \in\left[\frac{1}{n}, 1-\frac{1}{n}\right], x \in\left[\frac{1}{n}, R\right], x \in[-C, C]\right\}
$$

and define an open, bounded and convex set

$$
\Omega_{\bar{R}}=\left\{x \in C^{1}[0,1]:\|x\|_{1}<\bar{R}\right\} .
$$

For $x \in \bar{\Omega}_{\bar{R}}$, we have

$$
\begin{aligned}
&\left\|T_{n} x\right\| \leq \frac{1}{n}+\max _{t \in[0,1]}\left|\int_{1 / n}^{1-1 / n} G_{n}(t, s) F_{n}\left(s, x(s), x^{\prime}(s)\right) d s\right| \\
& \leq \frac{1}{3}+M_{1} \int_{1 / n}^{1-1 / n}\left|G_{n}(s, s)\right| d s=\frac{1}{3}+\frac{M_{1} \gamma^{2}}{6}, \\
&\left\|\left(T_{n} x\right)^{\prime}\right\| \leq \max _{t \in[0,1]}\left|\int_{1 / n}^{1-1 / n} \frac{\partial G_{n}}{\partial t}(t, s) F_{n}\left(s, x(s), x^{\prime}(s)\right) d s\right| \leq \frac{M_{1} \gamma}{2} .
\end{aligned}
$$

It follows that

$$
\left\|T_{n} x\right\|_{1}=\max \left\{\left\|T_{n} x\right\|, \frac{\gamma}{3}\left\|\left(T_{n} x\right)^{\prime}\right\|\right\} \leq \frac{1}{3}+\frac{M_{1} \gamma^{2}}{6}<\bar{R} \text { for every } x \in \bar{\Omega}_{\bar{R}} .
$$

Hence, $T_{n}\left(\bar{\Omega}_{\bar{R}}\right) \subset \Omega_{\bar{R}}$. Consequently, by Schauder's fixed point theorem, the BVP (2.1) has a solution in $\Omega_{\bar{R}}$.

Now, we show that any solution $x$ of $(2.1)$ must satisfies 2.2 . Firstly, we show that $x<R$ on $\left[\frac{1}{n}, 1-\frac{1}{n}\right]$. Assume that this is not true and $x(t) \geq R$ for some $t \in\left[\frac{1}{n}, 1-\frac{1}{n}\right]$. Let

$$
\xi=\min \left\{t \in\left[\frac{1}{n}, 1-\frac{1}{n}\right]: x(t)=R\right\} .
$$

We discuss different cases:

Case 1: If $\xi \leq 1 / 2$, since $x\left(\frac{1}{n}\right)=\frac{1}{n}<R$, there exist subintervals, say $\left[\xi_{2 i-1}, \xi_{2 i}\right] \subseteq$ $\left[\frac{1}{n}, \xi\right], i=1,2,3, \cdots, m$ such that

(1): $\xi_{1}=\frac{1}{n}, \xi_{2 m}=\xi, \xi_{2 i-1}<\xi_{2 i}$ for $i=1,2,3, \cdots, m$,

(2) $\xi_{2 i} \leq \xi_{2 i+1}, x\left(\xi_{2 i}\right)=x\left(\xi_{2 i+1}\right)$ and $x^{\prime}\left(\xi_{2 i}\right)=0$ for $i=1,2,3, \cdots, m-1$,

(3) $x^{\prime}(t) \geq 0$ for $t \in\left[\xi_{2 i-1}, \xi_{2 i}\right], i=1,2,3, \cdots, m$. 
For $t \in\left[\xi_{2 i-1}, \xi_{2 i}\right], i=1,2,3, \cdots, m$, using $\left(A_{2}\right)$ and the fact that $x(t) \in\left[\frac{1}{n}, R\right]$, we have

$$
-x^{\prime \prime}(t)=F_{n}\left(t, x(t), x^{\prime}(t)\right)=f\left(t, p_{n}(x(t)), q\left(x^{\prime}(t)\right)\right) \leq k(t) F(x(t)), t \in\left[\xi_{2 i-1}, \xi_{2 i}\right] .
$$

Integrating (2.5) from $t$ to $\xi_{2 i}$, using (2) and the decreasing property of $F$, we obtain

$$
x^{\prime}(t) \leq F(x(t)) \int_{t}^{\xi_{2 i}} k(s) d s, t \in\left[\xi_{2 i-1}, \xi_{2 i}\right], i=1,2,3, \cdots, m,
$$

which implies that

$$
\frac{x^{\prime}(t)}{F(x(t))} \leq \int_{t}^{\xi_{2 i}} k(s) d s, t \in\left[\xi_{2 i-1}, \xi_{2 i}\right], i=1,2,3, \cdots, m .
$$

Integrating (2.6) from $\xi_{2 i-1}$ to $\xi_{2 i}$, we have

$$
\int_{\xi_{2 i-1}}^{\xi_{2 i}} \frac{x^{\prime}(t)}{F(x(t))} d t \leq \int_{\xi_{2 i-1}}^{\xi_{2 i}} \int_{t}^{\xi_{2 i}} k(s) d s d t
$$

which can be written as

$$
\int_{x\left(\xi_{2 i-1}\right)}^{x\left(\xi_{2 i}\right)} \frac{d u}{F(u)} \leq \int_{\xi_{2 i-1}}^{\xi_{2 i}} s k(s) d s, i=1,2,3, \cdots, m .
$$

Summing from $i=1$ to $m$ and using $(2)\left(x\left(\xi_{2 i}\right)=x\left(\xi_{2 i+1}\right)\right)$, we obtain

$$
\int_{1 / n}^{R} \frac{d u}{F(u)} \leq \int_{0}^{1 / 2} \operatorname{sk}(s) d s .
$$

Letting $n \rightarrow \infty$, we have

$$
\int_{0}^{R} \frac{d u}{F(u)} \leq \int_{0}^{1 / 2} s k(s) d s
$$

a contradiction to 1.2 .

Case 2: Let $\xi \geq 1 / 2$ and $\eta=\max \left\{t \in\left[\frac{1}{2}, 1-\frac{1}{n}\right]: x(t)=R\right\}$. Since $x\left(1-\frac{1}{n}\right)=$ $\frac{1}{n}<R$, there exist subintervals $\left[\eta_{2 i}, \eta_{2 i-1}\right] \subseteq\left[\frac{1}{2}, 1-\frac{1}{n}\right], i=1,2,3, \cdots, m^{\prime}$ such that

(4) $\eta_{1}=1-\frac{1}{n}, \eta_{2 m^{\prime}}=\eta, \eta_{2 i}<\eta_{2 i-1}$ for $i=1,2,3, \cdots, m^{\prime}$,

(5) $\eta_{2 i+1} \leq \eta_{2 i}, x\left(\eta_{2 i}\right)=x\left(\eta_{2 i+1}\right), x^{\prime}\left(\eta_{2 i}\right)=0$ for $i=1,2,3, \cdots, m^{\prime}-1$ and

(6) $x^{\prime}(t) \leq 0$ for $t \in\left[\eta_{2 i}, \eta_{2 i-1}\right], i=1,2,3, \cdots, m^{\prime}$.

Integrating (2.5) from $\eta_{2 i}$ to $t$, using (5), the decreasing property of $F$ and then integrating from $\eta_{2 i}$ to $\eta_{2 i-1}$, we obtain

$$
\int_{x\left(\eta_{2 i-1}\right)}^{x\left(\eta_{2 i}\right)} \frac{d u}{F(u)} \leq \int_{\eta_{2 i}}^{\eta_{2 i-1}}(1-s) k(s) d s, i=1,2,3, \cdots, m^{\prime} .
$$

Summing 2.8 from $i=1$ to $i=m^{\prime}$ and using (5) $\left(x\left(\eta_{2 i}\right)=x\left(\eta_{2 i+1}\right)\right)$, we obtain

$$
\int_{1 / n}^{R} \frac{d u}{F(u)} \leq \int_{1 / 2}^{1}(1-s) k(s) d s .
$$


Letting limit $n \rightarrow \infty$, we get

$$
\int_{0}^{R} \frac{d u}{F(u)} \leq \int_{1 / 2}^{1}(1-s) k(s) d s
$$

which is a contradiction to 1.2 .

Now we show that any solution $x(t)$ of 2.1 must satisfies $\left|x^{\prime}(t)\right| \leq C, t \in$ $\left[\frac{1}{n}, 1-\frac{1}{n}\right]$. From the boundary conditions, $x\left(\frac{1}{n}\right)=\frac{1}{n}$ and $x\left(1-\frac{1}{n}\right)=\frac{1}{n}$, it follows that there exist $p \in\left(\frac{1}{n}, 1-\frac{1}{n}\right)$ such that $x^{\prime}(p)=0$. Suppose there exist $t_{0} \in\left(\frac{1}{n}, 1-\frac{1}{n}\right)$ such that $x^{\prime}\left(t_{0}\right)>C$. As in the first part of this theorem, choose $\left[\xi_{2 i-1}, \xi_{2 i}\right] \subseteq\left[\frac{1}{n}, 1-\frac{1}{n}\right]$ such that

$$
x^{\prime}(t) \geq 0 \text { on }\left[\xi_{2 i-1}, \xi_{2 i}\right] \text { and } x^{\prime}\left(\xi_{2 i}\right)=0, i=1,2,3, \cdots .
$$

Hence, there exist some $i_{0}$ such that $t_{0} \in\left[\xi_{2 i_{0}-1}, \xi_{2 i_{0}}\right]$. Let

$$
C_{1}=\max \left\{x^{\prime}(t): t \in\left[\xi_{2 i_{0}-1}, \xi_{2 i_{0}}\right]\right\}=x^{\prime}\left(\xi^{*}\right) .
$$

Clearly, $C_{1} \geq C$ and in view of $\left(A_{2}\right)$, we have

$$
-x^{\prime \prime}(t)=f\left(t, x(t), q\left(x^{\prime}(t)\right)\right) \leq k(t) F(x(t)) \leq M .
$$

Hence,

$$
-x^{\prime}(t) x^{\prime \prime}(t) \leq M x^{\prime}(t), t \in\left[\xi_{2 i_{0}-1}, \xi_{2 i_{0}}\right] .
$$

Integrating from $\xi^{*}$ to $\xi_{2 i_{0}}$, we have

$$
-\int_{\xi^{*}}^{\xi_{2 i_{0}}} x^{\prime}(t) x^{\prime \prime}(t) d t \leq M \int_{\xi^{*}}^{\xi_{2 i_{0}}} x^{\prime}(t) d t,
$$

implies that

$$
\int_{0}^{C_{1}} v d v \leq M R \Rightarrow C_{1} \leq \sqrt{2 M R}
$$

which contradict the definition of $C$. Hence, $x^{\prime}(t) \leq C, t \in\left[\frac{1}{n}, 1-\frac{1}{n}\right]$.

Similarly, we can show that $x^{\prime}(t) \geq-C, t \in\left[\frac{1}{n}, 1-\frac{1}{n}\right]$.

Theorem 2.2. Assume that $\left(A_{1}\right)$ and $\left(A_{2}\right)$ hold. Then, the boundary value problem (1.1) has a positive solution $x$.

Proof. By Theorem 2.1, any solution $x_{n}$ of (1.3) satisfies

$$
\frac{1}{n} \leq x_{n}(t) \leq R,\left|x_{n}^{\prime}(t)\right| \leq C \text { for } t \in\left[\frac{1}{n}, 1-\frac{1}{n}\right], n=3,4,5, \ldots
$$

Hence, for each $h \in(0,1 / 2)$, there exist a natural number $m \in\{3,4,5, \cdots\}$ such that $x_{n}(t)>0$ for all $t \in[h, 1-h]$ and $n \geq m$.

Consider the integral equation,

$$
\begin{aligned}
x_{n}(t)= & \frac{x_{n}(1-h)-x_{n}(h)}{1-2 h}(t-h)+x_{n}(h)+\int_{h}^{t} \frac{(s-h)(1-h-t)}{1-2 h} f\left(s, x_{n}(s), x_{n}^{\prime}(s)\right) d s \\
& +\int_{t}^{1-h} \frac{(t-h)(1-h-s)}{1-2 h} f\left(s, x_{n}(s), x_{n}^{\prime}(s)\right) d s, t \in[h, 1-h] .
\end{aligned}
$$


Differentiating with respect to $t$, we obtain

$$
\begin{aligned}
x_{n}^{\prime}(t)= & \frac{x_{n}(1-h)-x_{n}(h)}{1-2 h}-\frac{1}{1-2 h} \int_{h}^{t}(s-h) f\left(s, x_{n}(s), x_{n}^{\prime}(s)\right) d s \\
& +\frac{1}{1-2 h} \int_{t}^{1-h}(1-h-s) f\left(s, x_{n}(s), x_{n}^{\prime}(s)\right) d s .
\end{aligned}
$$

For any $t_{1}, t_{2} \in[h, 1-h]$, we have

$$
\begin{aligned}
\left|x_{n}^{\prime}\left(t_{2}\right)-x_{n}^{\prime}\left(t_{1}\right)\right|= & \mid \frac{-1}{1-2 h} \int_{h}^{t_{2}}(s-h) f\left(s, x_{n}(s), x_{n}^{\prime}(s)\right) d s \\
& +\frac{1}{1-2 h} \int_{t_{2}}^{1-h}(1-h-s) f\left(s, x_{n}(s), x_{n}^{\prime}(s)\right) d s \\
& +\frac{1}{1-2 h} \int_{h}^{t_{1}}(s-h) f\left(s, x_{n}(s), x_{n}^{\prime}(s)\right) d s \\
& -\frac{1}{1-2 h} \int_{t_{1}}^{1-h}(1-h-s) f\left(s, x_{n}(s), x_{n}^{\prime}(s)\right) d s \mid \\
= & \frac{1}{1-2 h}\left|\int_{t_{1}}^{t_{2}}(s-h) f\left(s, x_{n}(s), x_{n}^{\prime}(s)\right) d s+\int_{t_{1}}^{t_{2}}(1-h-s) f\left(s, x_{n}(s), x_{n}^{\prime}(s)\right) d s\right| \\
\leq & L\left|t_{2}-t_{1}\right|,
\end{aligned}
$$

where $L=\max \left\{f(t, u, v):(t, u, v) \in[h, 1-h] \times\left[\frac{1}{n}, R\right] \times[-C, C]\right\}$. Thus the sequences $\left\{x_{n}\right\}$ and $\left\{x_{n}^{\prime}\right\}$ are uniformly bounded and equicontinuous. By ArzelàAscoli theorem, there exist a subsequence $\left\{x_{n_{k}}\right\}$ of $\left\{x_{n}\right\}$ converging uniformly on $[h, 1-h]$ such that

$$
\begin{aligned}
& \lim _{n_{k} \rightarrow \infty} x_{n_{k}}(t)=x(t), \\
& \lim _{n_{k} \rightarrow \infty} x_{n_{k}}^{\prime}(t)=x^{\prime}(t),
\end{aligned}
$$

where $x \in C^{1}[0,1]$. Taking $\lim _{h \rightarrow 0}$, we have

$$
\lim _{n_{k} \rightarrow \infty} x_{n_{k}}(t)=x(t) \text { on }(0,1) .
$$

Further, $x>0$ on $(0,1)$. Letting $\lim _{n_{k} \rightarrow \infty}, 2.12$ and 2.10 yield

$$
\begin{gathered}
x(t)=\frac{x(1-h)-x(h)}{1-2 h}(t-h)+x(h)+\frac{1}{1-2 h} \int_{h}^{t}(s-h)(1-h-t) f\left(s, x(s), x^{\prime}(s)\right) d s \\
+\frac{1}{1-2 h} \int_{t}^{1-h}(t-h)(1-h-s) f\left(s, x(s), x^{\prime}(s)\right) d s, \\
x^{\prime}(t)=\frac{x(1-h)-x(h)}{1-2 h}-\frac{1}{1-2 h} \int_{h}^{t}(s-h) f\left(s, x(s), x^{\prime}(s)\right) d s \\
+\frac{1}{1-2 h} \int_{t}^{1-h}(1-h-s) f\left(s, x(s), x^{\prime}(s)\right) d s .
\end{gathered}
$$


Hence,

$$
\begin{aligned}
& x^{\prime \prime}(t)=-\frac{1}{1-2 h}(t-h) f\left(t, x(t), x^{\prime}(t)\right)-\frac{1}{1-2 h}(1-h-t) f\left(t, x(t), x^{\prime}(t)\right)=-f\left(t, x(t), x^{\prime}(t)\right) \\
& \Rightarrow \quad-x^{\prime \prime}(t)=f\left(t, x(t), x^{\prime}(t)\right), t \in(0,1)
\end{aligned}
$$

which implies that $x$ satisfies the differential equation (1.1). Moreover,

$$
x(0)=\lim _{n_{k} \rightarrow \infty} x\left(\frac{1}{n_{k}}\right)=\lim _{n_{k} \rightarrow \infty} x_{n_{k}}\left(\frac{1}{n_{k}}\right)=\lim _{n_{k} \rightarrow \infty} \frac{1}{n_{k}}=0,
$$

and

$$
x(1)=\lim _{n_{k} \rightarrow \infty} x\left(1-\frac{1}{n_{k}}\right)=\lim _{n_{k} \rightarrow \infty} x_{n_{k}}\left(1-\frac{1}{n_{k}}\right)=\lim _{n_{k} \rightarrow \infty} \frac{1}{n_{k}}=0,
$$

which implies that $x$ also satisfies the boundary conditions and hence is a solution of (1.1).

Example 2.3. Consider the boundary value problem

$$
\begin{aligned}
-x^{\prime \prime}(t) & =\frac{x^{\prime}(t)+5}{t(1-t)(x(t))^{2}} ; t \in(0,1) \\
x(0) & =0, x(1)=0 .
\end{aligned}
$$

Choose $k(t)=\frac{1}{t(1-t)}$ and $F(u)=\frac{9}{u^{2}}$. Clearly $F$ is decreasing and $\int_{0}^{\infty} \frac{d u}{F(u)}=\infty$. Since

$$
\int_{0}^{\frac{1}{2}} t k(t) d t=\ln 2, \int_{\frac{1}{2}}^{1}(1-t) k(t) d t=\ln 2 .
$$

Hence, from the relation $\int_{1}^{R} \frac{d u}{F(u)}>\ln 2$, we have $R>(1+27 \ln 2)^{3}$. Also,

$$
M=\max \left\{F\left(\frac{1}{n}\right) k(t): t \in\left[\frac{1}{n}, 1-\frac{1}{n}\right]\right\}=\max \left\{\frac{9 n^{2}}{t(1-t)}: t \in\left[\frac{1}{n}, 1-\frac{1}{n}\right]\right\}=9 .
$$

Hence $C=3$. Moreover,

$0 \leq \frac{x^{\prime}(t)+5}{t^{\mu}(1-t)^{\nu}(x(t))^{2}}=f\left(t, x(t), x^{\prime}(t)\right) \leq k(t) F(x(t))$ for $x^{\prime}(t) \in[-3,3], t \in(0,1)$.

By Theorem 2.2, the problem 2.12 has a solution $x$ such that

$$
0<x(t) \leq(1+27 \ln 2)^{3},\left|x^{\prime}(t)\right| \leq 3, t \in(0,1) .
$$

Acknowledgements: Research of R. A. Khan is supported by HEC, Pakistan, Project 2-3(50)/PDFP/HEC/2008/1. 


\section{REFERENCES}

[1] G. A. Afrouzi, M. Khaleghy Moghaddam, J. Mohammadpour and M. Zameni, On the positive and negative solutions of Laplacian BVP with Neumann boundary conditions, J. Nonlinear Sci. Appl., 2 (2009), 38-45.

[2] R.P. Agarwal and D. O' Regan, Twin solutions to singular Dirichlet problems, J. Math. Anal. Appl., 240 (1999), 433-445. 1

[3] R.P. Agarwal and D. O' Regan, An upper and lower solution approach for a generalized Thomas-Fermi theory of neutral atoms, Mathematical Problems in Engineering, 8 (2002), $135-142$.

[4] R. P. Agarwal and D. O'Regan, Singular differential and integral equations with applications, Kluwer Academic Publishers, London, 2003. 1

[5] R. P. Agarwal, D. O'Regan and P. K. Palamides, The generalized Thomas-Fermi singular boundary value problems for neutral atoms, Math. Methods in the Appl. Sci., 29 (2006), $49-66$. 1

[6] M. van den Berg, P. Gilkey and R. Seeley, Heat content asymptotics with singular initial temperature distributions, J. Funct. Anal., 254 (2008), 3093-3122. 1

[7] A. Callegari and A. Nachman, A nonlinear singular boundary value problem in the theory of pseudoplastic fluids, SIAM J. Appl. Math., 38 (1980), 275-282. 1 .

[8] J. Chu and D. Franco, Non-collision periodic solutions of second order singular dynamical systems, J. Math. Anal. Appl., 344 (2008), 898-905. 1

[9] J. Chu and J.J. Nieto, Impulsive periodic solutions of first-order singular differential equations, Bull. London Math. Soc., 40 (2008), 143-150.1

[10] X. Du and Z. Zhao, Existence and uniqueness of positive solutions to a class of singular m-point boundary value problems, Appl. Math. Comput., 198 (2008), 487-493. 1

[11] M. Fenga and W. Gea, Positive solutions for a class of m-point singular boundary value problems, Math. Comput. Modelling, 46 (2007), 375-383. 1

[12] J. Janus and J. Myjak, A generalized Emden-Fowler equation with a negative exponent, Nonlinear Anal., 23 (1994), 953-970. 1

[13] R. A. Khan, Positive solutions of four point singular boundary value problems, Appl. Math. Comput., 201 (2008), 762-773. 1

[14] S. K. Ntouyas and P. K. Palamides, On Sturm-Liouville and Thomas-Fermi Singular Boundary Value Problems, Nonlinear Oscillations, 4 (2001), 326-344. 1

[15] A. Orpel, On the existence of bounded positive solutions for a class of singular BVPs, Nonlinear Anal., 69 (2008), 1389-1395. 1

[16] D. O'Regan, Singular differential equations with linear and nonlinear boundary conditions, Computers Math. Appl., 35 (1998), 81-97. 1

[17] J. V. Shin, A singular nonlinear differential equation arising in the Homann flow, J. Math. Anal. Appl., 212 (1997) 443-451. 1

[18] E. Soewono, K. Vajravelu and R. N. Mohapatra, Existence and nonuniqueness of solutions of a singular nonlinear boundary layer problem, J. Math. Anal. Appl., 159 (1991), 251-270. 1

[19] G.C. Yang, Existence of solutions to the third-order nonlinear differential equations arising in boundary layer theory, Appl. Math. Lett., 6 (2003),827- 832. 1

[20] G.C. Yang, Positive solutions of singular Dirichlet boundary value problems with signchanging nonlinearities, Comp. Math. Appl., 51 (2006), 1463-1470.1] 1

[21] Z. Yong, Positive solutions of singular sublinear Emden-Fower boundary value problems, J. Math. Anal. Appl., 185 (1994), 215-222. 1 
1

${ }^{1}$ Centre for Advanced Mathematics and Physics, National University of Sciences and Technology, Campus of College of Electrical and Mechanical Engineering, Peshawar Road, Rawalpindi, Pakistan

E-mail address: rahmat _ alipk@yahoo.com

${ }^{2}$ Centre for Advanced Mathematics and Physics, National University of Sciences and Technology, Campus of College of Electrical and Mechanical Engineering, Peshawar Road, Rawalpindi, Pakistan

E-mail address: naseerasif@yahoo.com 